\title{
Syntheses of Homochiral Multinuclear Ru Complexes Based on Oligomeric Bibenzimidazoles
}

\author{
Jun Yin and Ronald L. Elsenbaumer* \\ Department of Chemistry and Biochemistry, The University of Texas at Arlington \\ Arlington, TX 76019, USA \\ Elsenbaumer@uta.edu
}

Supporting Information

Table of Contents

Experimental Section (The general part)

COSY spectra of $\Lambda \Lambda-5$

S3

${ }^{13} \mathrm{C}-\mathrm{NMR}$ spectra of $\Lambda \Lambda-5$

S4

X-ray crystallography data of $\Lambda_{4}-\mathrm{Ru}-6$

S5-S41

\section{Experimental Section (The general part):}

Reagents and Materials

All reagents were used without further purification. 2,2'-Bipyridine (99\%), $\mathrm{RuCl}_{3}(98 \%)$ and ammonium hexafluorophosphate (95+\%) were purchased from Alfa. Dibenzoyl-L-tartaric acid (98\%) and tetra- $n$-butylammonium chloride (98\%) were purchased from Aldrich. Acetonitrile was distilled over $\mathrm{CaH}_{2}$ before use. Nylon membranes filter paper (pore size: $0.2 \mu \mathrm{m}$ or $0.45 \mu \mathrm{m}$ ) for the filtration of the Ru complexes was purchased from Cole-Parmer Instrument Co. 


\section{Measurements}

NMR spectra were obtained on a JEOL Eclipse+ $500 \mathrm{MHz}$ spectrometer. UV-vis spectra were measured using a Varian Cary 500 UV-vis-NIR spectrophotometer. Elemental analysis was performed by Quantitative Technologies Inc. (QTI) (Whitehouse, NJ). Mass analysis was performed by Scripps Research Institute (La Jolla, CA). MALDI-TOF spectra were obtained on Applied Biosystems Voyager STR mass spectrometer. Cyclic Voltammogram experiments were performed at $20{ }^{\circ} \mathrm{C}$ using a $\mathrm{PC}$ controlled potentiostat ( $\mathrm{CH}$ Instruments, electroanalytical analyzer). The working electrode was a 1.5 mm glassy carbon electrode and the auxiliary electrode was a platinum wire. The reference electrode was a no leak $\mathrm{Ag} / \mathrm{AgCl}$ reference electrode. They were purchased from Cypress Systems, Inc. (Lawrence, KS). Circular Dichroism spectra were recorded on a JASCO J-710 spectropolarimeter. 


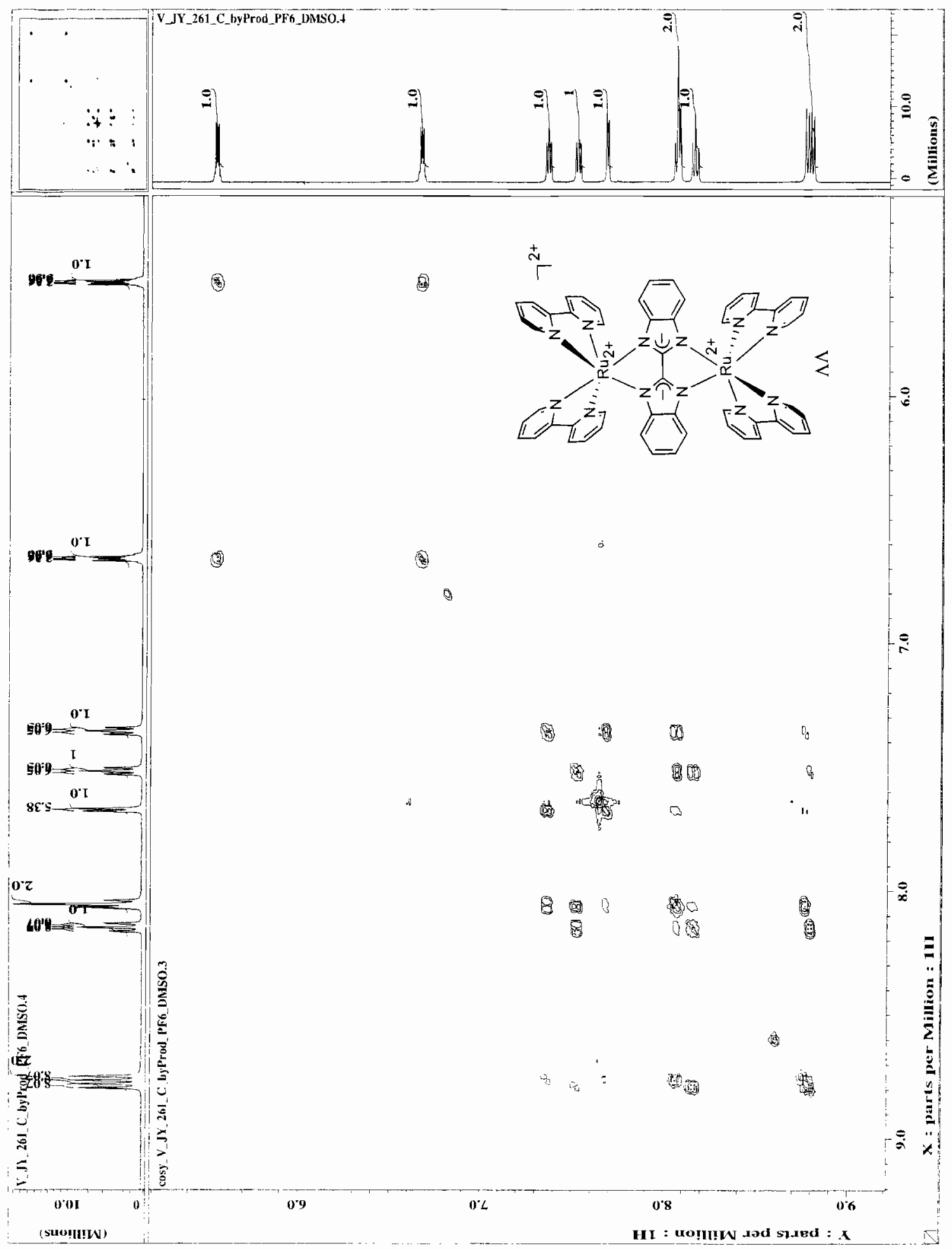




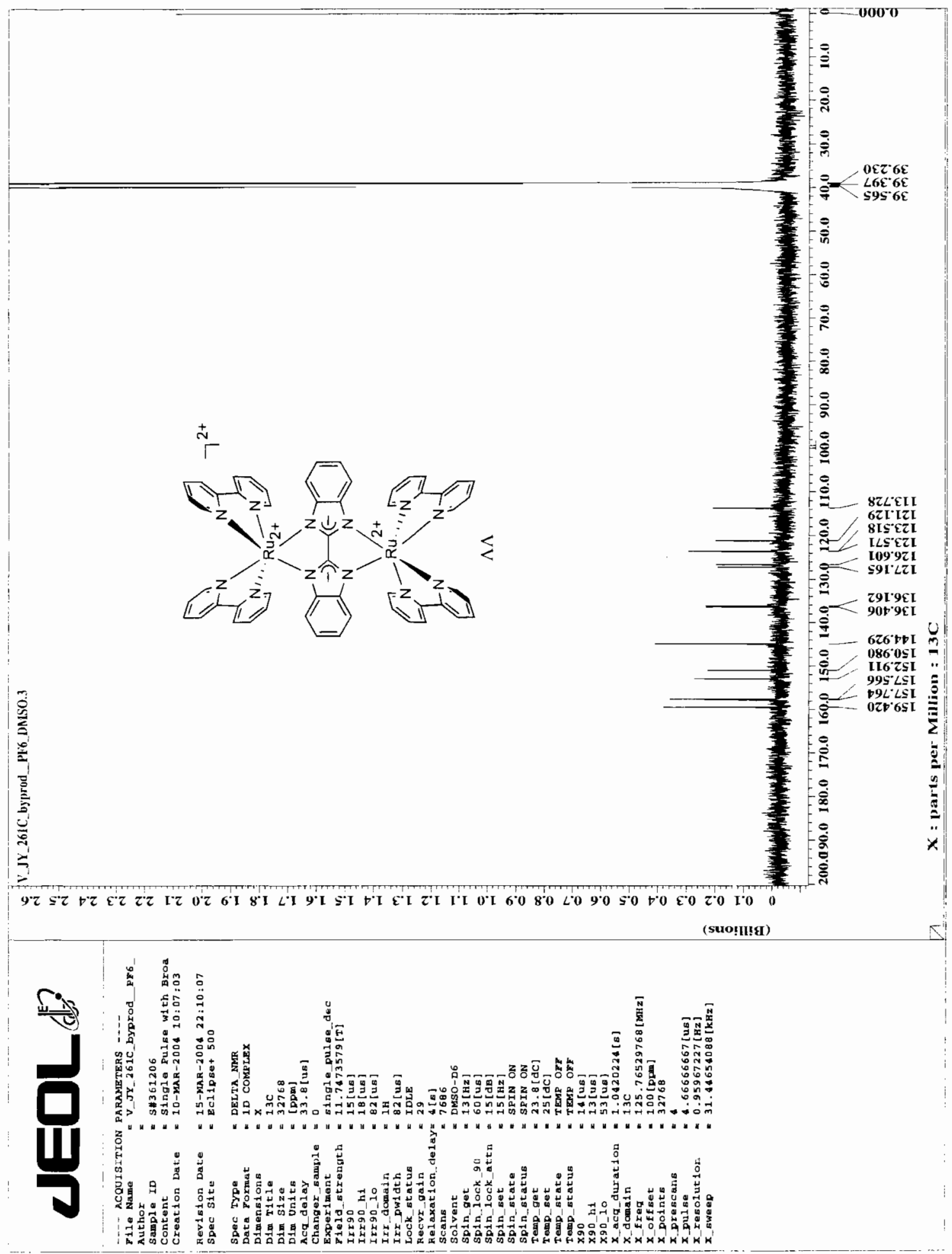




\section{X-ray Diffraction Laboratory \\ Center for Chemical Characterization and Analysis \\ Department of Chemistry \\ Texas A \& M University}

Structure Report for Ronald L. Elsenbaumer

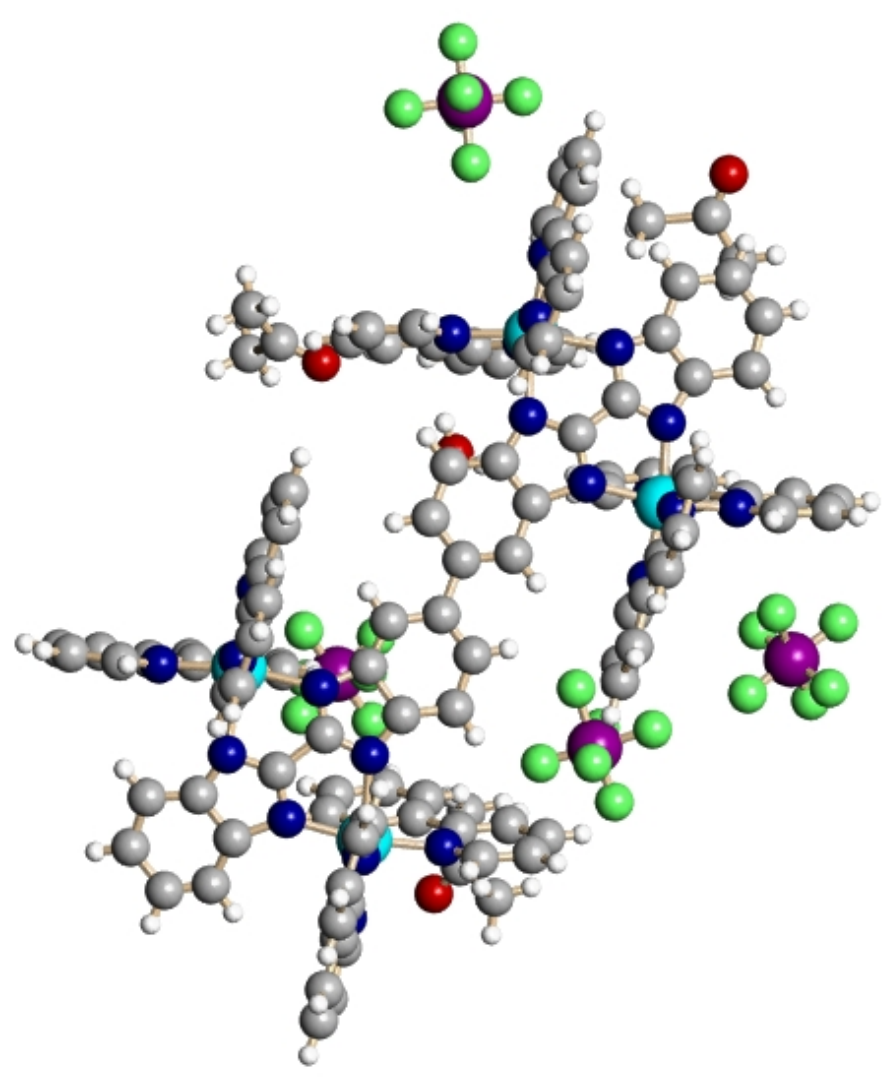

Project Name: UTA1

Date: March 23, 2006 

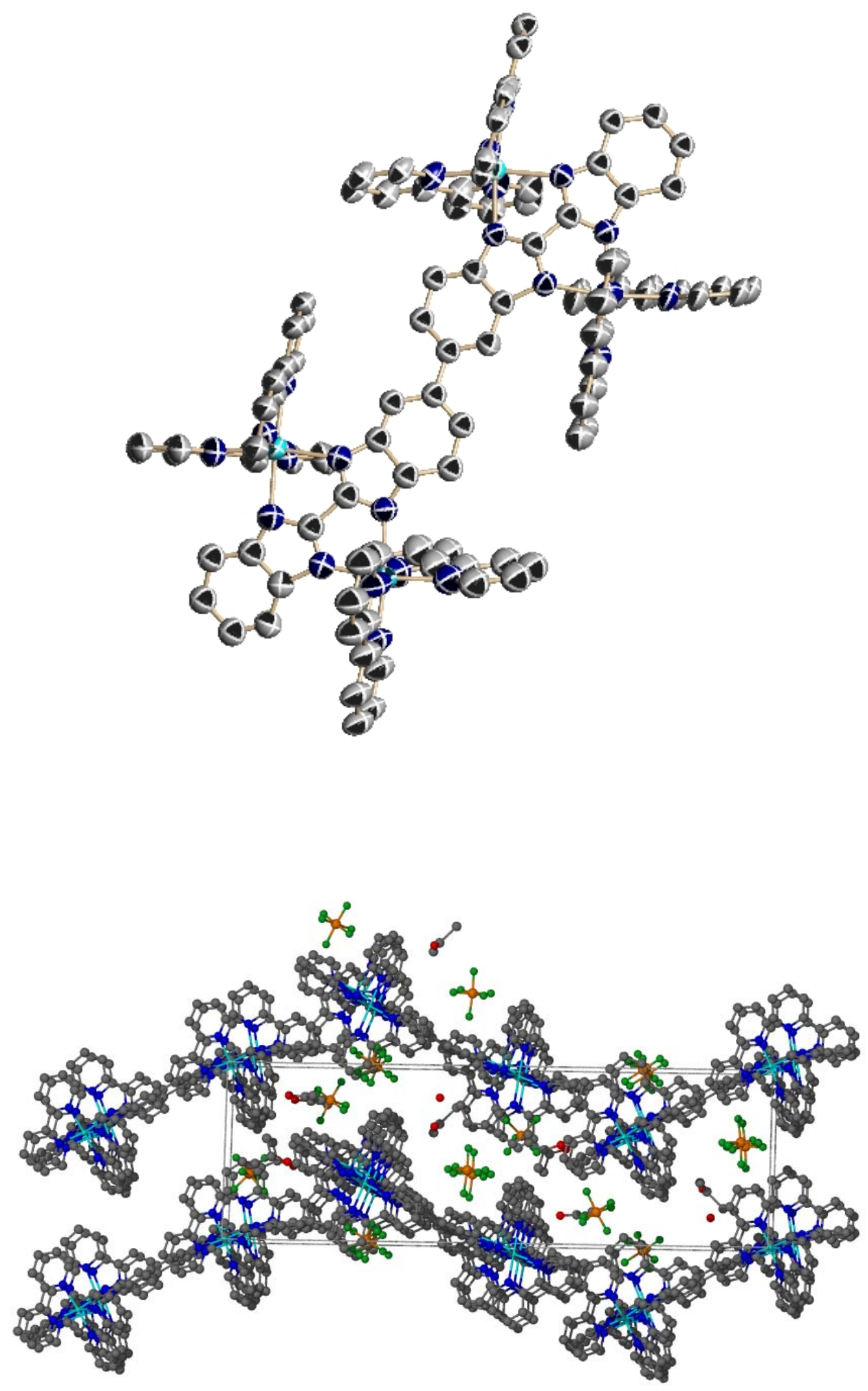


\section{Comments}

The sample was composed of very small weak scattering crystals. A full data set was collected however the high angle data was extremely weak and the intensity measurements at high angle are not as reliable as the low angle data. As a result the $R_{\text {int }}$ and the $R_{\text {sigma }}$ are relatively high. These values reflect the error associated with the high angle data. To obtain a chemical and structurally correct model, the 2,2'-dipyridyl, $\mathrm{PF}_{6}$ and acetone molecules were refined with restraints. The $\mathrm{Ru}$ and the bridging organic ligand were not restrained.

\section{Data Collection}

A Leica MZ7 polarizing microscope was used to identify a suitable specimen from a representative sampling of materials. The specimen was then fixed to a nylon loop which in turn was fashioned to a copper mounting pin. The mounted powder was then placed in a cold nitrogen stream (Oxford) maintained at $110 \mathrm{~K}$.

A BRUKER D8 GADDS general purpose three-circle X-ray diffractometer was employed for sample screening and data collection. The goniometer was controlled using the GADDS software suite (Microsoft Win 2000 operating system). The sample was optically centered with the aid of a video camera such that no translations were observed as the crystal was rotated through all positions. The detector was set at $5.0 \mathrm{~cm}$ from the crystal sample (MWPC Hi-Star Detector, 512x512 pixel). The X-ray radiation employed was generated from a Cu sealed X-ray tube $\left(\mathrm{K}_{\alpha}=1.54184 \AA\right.$ with a potential of $40 \mathrm{kV}$ and a current of $\left.40 \mathrm{~mA}\right)$ and filtered with a graphite monochromator in the parallel mode (175 $\mathrm{mm}$ collimator with $0.5 \mathrm{~mm}$ pinholes).

A rotation exposure was taken to determine crystal quality and the X-ray beam intersection with the detector. The beam intersection coordinates were compared to the configured coordinates and changes were made accordingly. The rotation exposure indicated acceptable crystal quality and the unit cell determination was undertaken. Sixty data frames were taken at widths of $0.5^{\circ}$ with an exposure time of 30 seconds. Over 200 reflections were centered and their positions were determined. These reflections were used in the auto-indexing procedure to determine the unit cell. A suitable cell was found and refined by nonlinear least squares and Bravais lattice procedures and reported here in Table S1. The unit cell was verified by examination of the hkl overlays on several frames of data, including zone photographs. No super-cell or erroneous reflections were observed.

After careful examination of the unit cell, a standard data collection procedure was initiated. This procedure consists of collection of one hemisphere of data collected using omega scans, involving the collection $25200.5^{\circ}$ frames at fixed angles for $\phi, 2 \theta$, and $\chi\left(2 \theta=-28^{\circ}, \chi=54.73^{\circ}, 2 \theta=-90^{\circ}, \chi=54.73^{\circ}\right)$, while varying omega. Addition data frames were collected to complete the data set. Each frame was exposed for $30 \mathrm{sec}$. The total data collection was performed for duration of approximately 24 hours at $110 \mathrm{~K}$. No significant intensity fluctuations of equivalent reflections were observed.

After data collection, the crystal was measured carefully for size, morphology and color. These measurements are reported in Table S1. 
Table S1. Crystal data and structure refinement.

Identification code

Empirical formula

Formula weight

Temperature

Wavelength

Crystal system

Space group

Unit cell dimensions

Volume

Z

Density (calculated)

Absorption coefficient

$\mathrm{F}(000)$

Crystal size

Theta range for data collection

Index ranges

Reflections collected

Independent reflections

Completeness to theta $=59.99^{\circ}$

Absorption correction

Max. and min. transmission

Refinement method ut

C112.50 H88 F24 N24 O2 P4 Ru4

2792.23

$110(2) \mathrm{K}$

$1.54184 \AA$

Monoclinic

P2(1)

$$
\begin{array}{ll}
\mathrm{a}=13.457(5) \AA & \alpha=90^{\circ} . \\
\mathrm{b}=38.583(13) \AA & \beta=116.578(11)^{\circ} . \\
\mathrm{c}=14.127(4) \AA & \gamma=90^{\circ} .
\end{array}
$$

$6560(4) \AA^{3}$

2

$1.414 \mathrm{Mg} / \mathrm{m}^{3}$

$4.891 \mathrm{~mm}^{-1}$

2798

$0.10 \times 0.01 \times 0.01 \mathrm{~mm}^{3}$

3.77 to $59.99^{\circ}$

$-15<=\mathrm{h}<=15,-43<=\mathrm{k}<=43,-15<=\mathrm{l}<=15$

49575

$18293[\mathrm{R}(\mathrm{int})=0.3885]$

$98.8 \%$

Empirical

0.9760 and 0.6405

Full-matrix least-squares on $\mathrm{F}^{2}$ 
Data / restraints / parameters

Goodness-of-fit on $\mathrm{F}^{2}$

Final R indices [I $>2 \operatorname{sigma}(\mathrm{I})]$

$\mathrm{R}$ indices (all data)

Absolute structure parameter

Largest diff. peak and hole
18293 / 1779 / 1363

1.040

$\mathrm{R} 1=0.0766, \mathrm{wR} 2=0.1343$

$\mathrm{R} 1=0.3270, \mathrm{wR} 2=0.2855$

$0.00(3)$

0.443 and -0.344 e. $\AA^{-3}$ 
Table S2. Atomic coordinates $\left(\mathrm{x} 10^{4}\right)$ and equivalent isotropic displacement parameters $\left(\AA^{2} \times 10^{3}\right)$ for ut. $U(e q)$ is defined as one third of the trace of the orthogonalized $U^{i j}$ tensor.

\begin{tabular}{|c|c|c|c|c|}
\hline & $\mathrm{x}$ & $\mathrm{y}$ & $\mathrm{z}$ & $\mathrm{U}(\mathrm{eq})$ \\
\hline $\mathrm{Ru}(1)$ & $4541(2)$ & $5394(2)$ & $9312(2)$ & $71(1)$ \\
\hline $\mathrm{Ru}(2)$ & $943(2)$ & $5036(2)$ & $9982(3)$ & $75(1)$ \\
\hline $\mathrm{Ru}(3)$ & $3610(2)$ & $2367(2)$ & $14038(2)$ & $48(1)$ \\
\hline $\mathrm{Ru}(4)$ & $7089(2)$ & $2651(2)$ & $13096(3)$ & $76(1)$ \\
\hline $\mathrm{N}(1)$ & $3850(20)$ & $4943(7)$ & $9780(20)$ & $68(2)$ \\
\hline $\mathrm{N}(2)$ & $3050(20)$ & $5593(7)$ & $9190(20)$ & $72(3)$ \\
\hline $\mathrm{N}(3)$ & $1450(20)$ & $5493(7)$ & $9330(20)$ & $72(3)$ \\
\hline $\mathrm{N}(4)$ & $2360(20)$ & $4795(7)$ & $9920(20)$ & $66(2)$ \\
\hline $\mathrm{N}(5)$ & $4060(20)$ & $2751(7)$ & $13190(20)$ & $55(2)$ \\
\hline $\mathrm{N}(6)$ & $5120(20)$ & 2099(7) & $14180(20)$ & $58(2)$ \\
\hline $\mathrm{N}(7)$ & $6520(20)$ & $2249(7)$ & 13711(19) & $60(2)$ \\
\hline $\mathrm{N}(8)$ & $5560(20)$ & $2833(6)$ & $12830(20)$ & $56(2)$ \\
\hline $\mathrm{C}(1)$ & $2380(30)$ & $5910(5)$ & $9020(30)$ & $74(3)$ \\
\hline$C(2)$ & $2600(30)$ & $6264(9)$ & $8980(30)$ & $76(3)$ \\
\hline$C(3)$ & $1910(30)$ & $6487(9)$ & $8820(30)$ & $77(4)$ \\
\hline$C(4)$ & $1020(30)$ & $6409(9)$ & $9140(30)$ & $77(3)$ \\
\hline$C(5)$ & $730(30)$ & $6058(8)$ & $9200(30)$ & $76(3)$ \\
\hline$C(6)$ & $1450(20)$ & $5812(7)$ & $9210(30)$ & $74(3)$ \\
\hline$C(7)$ & $2430(30)$ & $5382(6)$ & $9310(30)$ & $71(2)$ \\
\hline$C(8)$ & $2920(20)$ & $5016(6)$ & $9770(30)$ & $68(2)$ \\
\hline
\end{tabular}




\begin{tabular}{|c|c|c|c|c|}
\hline $\mathrm{C}(9)$ & $3080(30)$ & $4521(6)$ & $10350(30)$ & $65(2)$ \\
\hline$C(10)$ & $3040(30)$ & $4225(8)$ & $10770(20)$ & $62(3)$ \\
\hline $\mathrm{C}(11)$ & $3990(30)$ & 4003(9) & $11120(30)$ & $60(3)$ \\
\hline $\mathrm{C}(12)$ & $4860(30)$ & $4085(8)$ & $10980(30)$ & $63(3)$ \\
\hline $\mathrm{C}(13)$ & $4970(30)$ & $4419(9)$ & $10530(30)$ & $65(3)$ \\
\hline$C(14)$ & $3950(20)$ & $4606(7)$ & $10060(30)$ & $66(2)$ \\
\hline$C(15)$ & $4770(30)$ & $3140(5)$ & $12500(20)$ & $56(2)$ \\
\hline$C(16)$ & $4800(30)$ & $3443(8)$ & $11900(20)$ & $57(3)$ \\
\hline $\mathrm{C}(17)$ & $3980(30)$ & $3662(9)$ & $11710(30)$ & $58(3)$ \\
\hline $\mathrm{C}(18)$ & $3160(30)$ & $3597(8)$ & $12040(30)$ & $59(3)$ \\
\hline $\mathrm{C}(19)$ & $3120(30)$ & $3297(8)$ & $12540(30)$ & $57(3)$ \\
\hline$C(20)$ & $3910(20)$ & $3069(7)$ & $12730(20)$ & $56(2)$ \\
\hline $\mathrm{C}(21)$ & $4960(20)$ & $2635(6)$ & $13130(20)$ & $56(2)$ \\
\hline$C(22)$ & $5560(20)$ & $2315(7)$ & $13660(20)$ & $59(2)$ \\
\hline$C(23)$ & $5920(30)$ & $1875(7)$ & $14600(20)$ & $60(2)$ \\
\hline $\mathrm{C}(24)$ & $5920(30)$ & $1569(8)$ & $15170(20)$ & $59(3)$ \\
\hline$C(25)$ & $6860(20)$ & $1362(9)$ & $15600(20)$ & $59(3)$ \\
\hline$C(26)$ & $7740(30)$ & $1438(8)$ & $15470(20)$ & $59(3)$ \\
\hline $\mathrm{C}(27)$ & $7750(30)$ & $1705(8)$ & $14730(20)$ & $60(3)$ \\
\hline $\mathrm{C}(28)$ & $6790(20)$ & 1941(7) & $14380(20)$ & $60(2)$ \\
\hline $\mathrm{O}(1 \mathrm{~W})$ & $6580(50)$ & $3881(12)$ & $8260(30)$ & $130(30)$ \\
\hline N11 & $5399(14)$ & $5487(4)$ & $10917(14)$ & $105(4)$ \\
\hline N21 & $6017(14)$ & $5134(4)$ & $9656(13)$ & $103(4)$ \\
\hline C11 & $5044(17)$ & $5662(5)$ & $11513(18)$ & $105(4)$ \\
\hline C21 & $5580(19)$ & $5653(5)$ & $12588(19)$ & $106(4)$ \\
\hline
\end{tabular}




\begin{tabular}{|c|c|c|c|c|}
\hline $\mathrm{C} 31$ & $6521(19)$ & $5460(5)$ & 13071(17) & $108(4)$ \\
\hline $\mathrm{C} 41$ & $6889(15)$ & $5277(5)$ & $12459(14)$ & $108(4)$ \\
\hline C51 & $6310(11)$ & $5293(4)$ & $11380(12)$ & $106(4)$ \\
\hline C61 & $6661(11)$ & $5101(4)$ & $10685(12)$ & $105(4)$ \\
\hline C71 & $7582(14)$ & $4897(5)$ & $11062(15)$ & $105(4)$ \\
\hline C81 & $7858(18)$ & $4725(5)$ & $10368(19)$ & $104(4)$ \\
\hline C91 & $7200(19)$ & $4757(5)$ & $9310(20)$ & $104(4)$ \\
\hline $\mathrm{C} 101$ & $6284(18)$ & $4964(5)$ & $8988(17)$ & $103(4)$ \\
\hline $\mathrm{N} 12$ & $4968(12)$ & $5848(4)$ & $8812(12)$ & $87(4)$ \\
\hline $\mathrm{N} 22$ & $3725(13)$ & $5310(4)$ & $7728(13)$ & $99(4)$ \\
\hline $\mathrm{C} 12$ & $5617(15)$ & $6106(5)$ & $9385(15)$ & $87(4)$ \\
\hline $\mathrm{C} 22$ & $5768(15)$ & $6403(5)$ & $8934(17)$ & $86(4)$ \\
\hline $\mathrm{C} 32$ & $5224(16)$ & $6438(5)$ & $7855(16)$ & $88(4)$ \\
\hline C42 & $4556(14)$ & $6171(4)$ & $7256(13)$ & $89(4)$ \\
\hline C52 & $4445(9)$ & $5877(4)$ & $7754(11)$ & $91(4)$ \\
\hline C62 & $3747(10)$ & $5581(4)$ & $7153(11)$ & $96(4)$ \\
\hline $\mathrm{C} 72$ & $3161(15)$ & $5581(5)$ & $6071(13)$ & $100(4)$ \\
\hline $\mathrm{C} 82$ & $2529(17)$ & $5296(6)$ & $5565(16)$ & $102(5)$ \\
\hline C92 & $2507(17)$ & $5017(6)$ & $6150(18)$ & $103(5)$ \\
\hline $\mathrm{C} 102$ & $3118(16)$ & $5033(5)$ & $7232(16)$ & $102(5)$ \\
\hline N13 & $-84(12)$ & $4816(4)$ & $8547(14)$ & $98(4)$ \\
\hline $\mathrm{N} 23$ & $527(12)$ & $4580(4)$ & $10494(13)$ & $91(4)$ \\
\hline $\mathrm{C} 13$ & $-349(15)$ & $4942(5)$ & $7594(17)$ & $98(4)$ \\
\hline $\mathrm{C} 23$ & $-894(15)$ & $4752(6)$ & $6700(18)$ & $100(4)$ \\
\hline $\mathrm{C} 33$ & $-1188(16)$ & $4421(6)$ & $6785(17)$ & $100(4)$ \\
\hline
\end{tabular}




\begin{tabular}{|c|c|c|c|c|}
\hline $\mathrm{C} 43$ & $-911(14)$ & $4288(5)$ & $7768(14)$ & $98(4)$ \\
\hline C53 & $-352(9)$ & $4490(4)$ & $8642(12)$ & $96(4)$ \\
\hline C63 & $-27(9)$ & $4361(4)$ & $9716(12)$ & $93(4)$ \\
\hline $\mathrm{C} 73$ & $-263(14)$ & $4034(4)$ & $9916(14)$ & $93(4)$ \\
\hline $\mathrm{C} 83$ & $67(16)$ & $3931(5)$ & $10936(17)$ & $95(4)$ \\
\hline C93 & $637(15)$ & $4155(5)$ & $11735(18)$ & $94(4)$ \\
\hline $\mathrm{C} 103$ & $852(15)$ & $4478(5)$ & $11482(15)$ & $92(4)$ \\
\hline N14 & $-228(12)$ & $5368(4)$ & $10086(13)$ & $96(4)$ \\
\hline $\mathrm{N} 24$ & $1865(12)$ & $5204(4)$ & $11425(13)$ & $98(4)$ \\
\hline $\mathrm{C} 14$ & $-1274(15)$ & $5436(5)$ & $9397(16)$ & $97(5)$ \\
\hline $\mathrm{C} 24$ & $-1958(17)$ & $5636(5)$ & $9653(18)$ & $97(5)$ \\
\hline C34 & $-1550(17)$ & $5775(5)$ & $10647(17)$ & $97(5)$ \\
\hline C44 & $-470(14)$ & $5704(5)$ & $11366(14)$ & $97(4)$ \\
\hline C54 & $176(11)$ & $5497(3)$ & $11067(11)$ & $97(4)$ \\
\hline C64 & $1337(11)$ & $5410(3)$ & $11806(11)$ & $99(4)$ \\
\hline $\mathrm{C} 74$ & $1847(13)$ & $5530(5)$ & $12825(13)$ & $100(4)$ \\
\hline C84 & $2929(16)$ & $5438(5)$ & $13468(16)$ & $100(5)$ \\
\hline C94 & $3473(16)$ & $5226(5)$ & $13082(18)$ & $100(5)$ \\
\hline C104 & 2911(15) & $5113(5)$ & $12055(17)$ & $99(5)$ \\
\hline N15 & 2392(11) & $2704(4)$ & $13995(10)$ & $66(4)$ \\
\hline $\mathrm{N} 25$ & $4437(11)$ & $2623(4)$ & $15436(10)$ & $72(4)$ \\
\hline $\mathrm{C} 15$ & 1371(13) & $2730(5)$ & $13268(12)$ & $64(4)$ \\
\hline $\mathrm{C} 25$ & $669(15)$ & $2966(5)$ & $13312(13)$ & $68(4)$ \\
\hline $\mathrm{C} 35$ & $1030(14)$ & $3187(5)$ & $14124(13)$ & $70(4)$ \\
\hline $\mathrm{C} 45$ & $2084(12)$ & $3161(4)$ & $14882(11)$ & $70(4)$ \\
\hline
\end{tabular}




\begin{tabular}{|c|c|c|c|c|}
\hline C55 & $2750(10)$ & 2916(3) & $14803(8)$ & $70(3)$ \\
\hline C65 & $3884(10)$ & $2874(3)$ & $15595(8)$ & $73(4)$ \\
\hline $\mathrm{C} 75$ & $4349(12)$ & $3076(4)$ & $16450(11)$ & $76(4)$ \\
\hline $\mathrm{C} 85$ & $5408(15)$ & $3023(5)$ & $17155(13)$ & $78(4)$ \\
\hline C95 & $5976(15)$ & $2766(5)$ & $16997(14)$ & $77(4)$ \\
\hline $\mathrm{C} 105$ & $5461(13)$ & $2571(5)$ & $16127(13)$ & $75(4)$ \\
\hline N16 & $3254(10)$ & $1928(4)$ & $14686(9)$ & $54(3)$ \\
\hline $\mathrm{N} 26$ & $2648(10)$ & $2089(4)$ & $12662(9)$ & $54(3)$ \\
\hline $\mathrm{C} 16$ & $3547(12)$ & $1862(4)$ & $15701(11)$ & $57(4)$ \\
\hline $\mathrm{C} 26$ & $3391(12)$ & $1546(5)$ & $16046(13)$ & $60(4)$ \\
\hline $\mathrm{C} 36$ & $2928(13)$ & $1285(5)$ & $15325(12)$ & $63(4)$ \\
\hline $\mathrm{C} 46$ & $2616(11)$ & 1351(4) & $14272(10)$ & $60(3)$ \\
\hline C56 & 2784(7) & 1677(3) & $13970(8)$ & $56(3)$ \\
\hline C66 & $2460(7)$ & 1763(3) & $12852(8)$ & $55(3)$ \\
\hline C76 & $1984(11)$ & $1528(4)$ & $12049(10)$ & $54(3)$ \\
\hline $\mathrm{C} 86$ & $1703(13)$ & $1628(4)$ & $11027(12)$ & $56(4)$ \\
\hline C96 & $1892(12)$ & 1961(5) & $10829(13)$ & $54(4)$ \\
\hline C106 & $2366(12)$ & $2184(4)$ & $11669(11)$ & $54(3)$ \\
\hline N17 & $7811(11)$ & $2854(5)$ & $14655(12)$ & $96(4)$ \\
\hline $\mathrm{N} 27$ & $8602(11)$ & $2426(4)$ & $13652(12)$ & $89(4)$ \\
\hline $\mathrm{C} 17$ & $7372(14)$ & $3063(5)$ & $15124(16)$ & $96(4)$ \\
\hline $\mathrm{C} 27$ & $7899(16)$ & $3133(5)$ & $16189(18)$ & $98(4)$ \\
\hline $\mathrm{C} 37$ & $8920(16)$ & $2986(6)$ & $16796(16)$ & $99(4)$ \\
\hline $\mathrm{C} 47$ & $9377(13)$ & $2768(5)$ & $16318(13)$ & $98(4)$ \\
\hline $\mathrm{C} 57$ & $8803(9)$ & $2704(4)$ & $15243(10)$ & $5(4)$ \\
\hline
\end{tabular}




\begin{tabular}{|c|c|c|c|c|}
\hline C67 & $9248(9)$ & $2470(4)$ & $14689(10)$ & $91(4)$ \\
\hline $\mathrm{C} 77$ & $10256(12)$ & $2305(5)$ & $15199(12)$ & $90(4)$ \\
\hline $\mathrm{C} 87$ & $10616(14)$ & 2091(5) & $14632(15)$ & $88(4)$ \\
\hline C97 & $9956(15)$ & $2044(5)$ & $13571(17)$ & $88(4)$ \\
\hline $\mathrm{C} 107$ & $8952(14)$ & $2216(5)$ & $13109(15)$ & $89(4)$ \\
\hline N18 & $7390(12)$ & $3074(4)$ & $12424(11)$ & $85(4)$ \\
\hline $\mathrm{N} 28$ & $6449(12)$ & $2455(4)$ & $11603(11)$ & $86(4)$ \\
\hline $\mathrm{C} 18$ & 7891(15) & $3375(5)$ & $12873(15)$ & $87(4)$ \\
\hline $\mathrm{C} 28$ & $7973(15)$ & $3651(5)$ & $12282(16)$ & $87(4)$ \\
\hline $\mathrm{C} 38$ & $7515(16)$ & $3618(5)$ & 11191(15) & $88(4)$ \\
\hline $\mathrm{C} 48$ & $7002(14)$ & $3307(4)$ & $10721(12)$ & $88(4)$ \\
\hline C58 & 6954(9) & $3037(4)$ & $11357(10)$ & $87(4)$ \\
\hline C68 & 6422(9) & 2696(4) & $10900(10)$ & $88(4)$ \\
\hline $\mathrm{C} 78$ & $5937(14)$ & $2627(5)$ & $9823(12)$ & $92(4)$ \\
\hline C88 & $5458(16)$ & $2304(5)$ & $9455(15)$ & $93(4)$ \\
\hline C98 & $5486(15)$ & $2056(5)$ & $10172(16)$ & $91(4)$ \\
\hline $\mathrm{C} 108$ & $5992(15)$ & $2141(5)$ & $11242(14)$ & $88(4)$ \\
\hline P19 & $9765(12)$ & $4450(5)$ & $14102(10)$ & $146(6)$ \\
\hline F19 & $9120(20)$ & $4394(6)$ & $12798(14)$ & $155(9)$ \\
\hline F29 & $10370(20)$ & $4465(7)$ & $15325(15)$ & 166(9) \\
\hline F39 & $10650(20)$ & $4660(8)$ & 13953(19) & $207(11)$ \\
\hline F49 & $8890(20)$ & $4221(8)$ & $14310(20)$ & $200(11)$ \\
\hline F59 & $10420(20)$ & $4105(6)$ & $14130(20)$ & 192(10) \\
\hline F69 & $9180(20)$ & $4779(7)$ & $14126(18)$ & $214(12)$ \\
\hline P110 & $2226(12)$ & $1817(4)$ & $18114(9)$ & $125(5)$ \\
\hline
\end{tabular}




\begin{tabular}{|c|c|c|c|c|}
\hline F110 & $1400(20)$ & $1640(6)$ & $16967(15)$ & 163(9) \\
\hline F210 & $2970(20)$ & 2017(6) & $19142(14)$ & 159(9) \\
\hline F310 & $1740(20)$ & 1581(6) & $18679(17)$ & $158(8)$ \\
\hline F410 & $2752(19)$ & 2079(6) & $17569(17)$ & 141(7) \\
\hline F510 & $1266(18)$ & 2094(6) & $17863(17)$ & 141(8) \\
\hline F610 & $3170(20)$ & $1569(7)$ & $18362(19)$ & $190(10)$ \\
\hline P111 & $6319(10)$ & 5391(4) & $6167(10)$ & $132(5)$ \\
\hline F111 & $6520(30)$ & $5670(8)$ & $7130(20)$ & $228(13)$ \\
\hline F211 & $6180(20)$ & $5144(6)$ & $5258(19)$ & 163(9) \\
\hline F311 & $6950(20)$ & 5114(7) & $7015(19)$ & $169(9)$ \\
\hline F411 & $5720(20)$ & $5672(6)$ & $5255(18)$ & $159(9)$ \\
\hline F511 & $7467(17)$ & $5526(6)$ & $6260(20)$ & $170(10)$ \\
\hline F611 & $5220(18)$ & $5274(7)$ & $6070(20)$ & $184(10)$ \\
\hline P112 & $-884(15)$ & $2627(5)$ & $10109(12)$ & 207(9) \\
\hline F112 & $-2000(20)$ & $2371(10)$ & $9610(30)$ & $295(15)$ \\
\hline F212 & $210(20)$ & 2833(8) & $10570(30)$ & $243(12)$ \\
\hline F312 & $-1190(20)$ & $2714(10)$ & $11010(20)$ & $263(14)$ \\
\hline F412 & $-520(30)$ & 2521(9) & $9210(20)$ & $280(15)$ \\
\hline F512 & $-240(20)$ & $2298(7)$ & $10760(20)$ & $224(12)$ \\
\hline F612 & $-1480(30)$ & 2940(9) & $9490(30)$ & $326(16)$ \\
\hline O113 & $12499(19)$ & $3750(6)$ & $16710(20)$ & $42(6)$ \\
\hline C113 & $10600(20)$ & $3715(11)$ & $16390(30)$ & $45(7)$ \\
\hline C213 & $11730(30)$ & $3835(5)$ & $16870(20)$ & $43(6)$ \\
\hline C313 & $12000(40)$ & $4147(11)$ & $17810(40)$ & $44(8)$ \\
\hline O114 & $-2860(30)$ & $1112(8)$ & $8235(18)$ & $114(9)$ \\
\hline
\end{tabular}




$\begin{array}{lrrrr}\text { C114 } & -1660(30) & 1559(14) & 8180(50) & 116(10) \\ \text { C214 } & -2640(30) & 1411(12) & 8160(20) & 115(9) \\ \text { C314 } & -3650(50) & 1704(14) & 8000(60) & 117(10) \\ \text { O115 } & 150(20) & 6151(11) & 5590(30) & 112(9) \\ \text { C115 } & -320(60) & 5562(13) & 5720(50) & 112(10) \\ \text { C215 } & 20(30) & 5852(14) & 5310(30) & 111(9) \\ \text { C315 } & 280(70) & 5758(17) & 4270(50) & 107(10)\end{array}$


Table S3. Bond lengths $[\AA]$ and angles $\left[^{\circ}\right]$.

\begin{tabular}{|c|c|}
\hline $\mathrm{Ru}(1)-\mathrm{N} 22$ & $2.031(16)$ \\
\hline $\mathrm{Ru}(1)-\mathrm{N} 11$ & $2.064(18)$ \\
\hline $\mathrm{Ru}(1)-\mathrm{N} 12$ & $2.064(15)$ \\
\hline $\mathrm{Ru}(1)-\mathrm{N} 21$ & $2.081(18)$ \\
\hline $\mathrm{Ru}(1)-\mathrm{N}(2)$ & $2.08(3)$ \\
\hline $\mathrm{Ru}(1)-\mathrm{N}(1)$ & $2.20(3)$ \\
\hline $\mathrm{Ru}(2)-\mathrm{N} 24$ & $1.962(17)$ \\
\hline $\mathrm{Ru}(2)-\mathrm{N} 13$ & $2.057(18)$ \\
\hline $\mathrm{Ru}(2)-\mathrm{N} 23$ & $2.070(14)$ \\
\hline $\mathrm{Ru}(2)-\mathrm{N} 14$ & $2.086(16)$ \\
\hline $\mathrm{Ru}(2)-\mathrm{N}(4)$ & $2.16(3)$ \\
\hline $\mathrm{Ru}(2)-\mathrm{N}(3)$ & $2.24(3)$ \\
\hline $\mathrm{Ru}(3)-\mathrm{N} 25$ & $2.038(13)$ \\
\hline $\mathrm{Ru}(3)-\mathrm{N} 15$ & $2.073(13)$ \\
\hline $\mathrm{Ru}(3)-\mathrm{N} 16$ & $2.079(12)$ \\
\hline $\mathrm{Ru}(3)-\mathrm{N} 26$ & $2.086(11)$ \\
\hline $\mathrm{Ru}(3)-\mathrm{N}(5)$ & $2.16(2)$ \\
\hline $\mathrm{Ru}(3)-\mathrm{N}(6)$ & $2.20(3)$ \\
\hline $\mathrm{Ru}(4)-\mathrm{N} 18$ & $2.018(14)$ \\
\hline $\mathrm{Ru}(4)-\mathrm{N} 27$ & $2.022(14)$ \\
\hline $\mathrm{Ru}(4)-\mathrm{N} 28$ & $2.034(15)$ \\
\hline $\mathrm{Ru}(4)-\mathrm{N}(8)$ & $2.05(2)$ \\
\hline $\mathrm{Ru}(4)-\mathrm{N}(7)$ & $2.08(2)$ \\
\hline
\end{tabular}




\begin{tabular}{|c|c|}
\hline $\mathrm{Ru}(4)-\mathrm{N} 17$ & $2.118(17)$ \\
\hline $\mathrm{N}(1)-\mathrm{C}(8)$ & $1.29(3)$ \\
\hline $\mathrm{N}(1)-\mathrm{C}(14)$ & $1.35(3)$ \\
\hline $\mathrm{N}(2)-\mathrm{C}(7)$ & $1.23(3)$ \\
\hline $\mathrm{N}(2)-\mathrm{C}(1)$ & $1.47(3)$ \\
\hline $\mathrm{N}(3)-\mathrm{C}(6)$ & $1.24(3)$ \\
\hline $\mathrm{N}(3)-\mathrm{C}(7)$ & $1.39(4)$ \\
\hline $\mathrm{N}(4)-\mathrm{C}(8)$ & $1.22(3)$ \\
\hline $\mathrm{N}(4)-\mathrm{C}(9)$ & $1.38(3)$ \\
\hline $\mathrm{N}(5)-\mathrm{C}(21)$ & $1.33(3)$ \\
\hline $\mathrm{N}(5)-\mathrm{C}(20)$ & $1.36(3)$ \\
\hline $\mathrm{N}(6)-\mathrm{C}(23)$ & $1.30(3)$ \\
\hline $\mathrm{N}(6)-\mathrm{C}(22)$ & $1.41(3)$ \\
\hline $\mathrm{N}(7)-\mathrm{C}(22)$ & $1.29(3)$ \\
\hline $\mathrm{N}(7)-\mathrm{C}(28)$ & $1.46(3)$ \\
\hline $\mathrm{N}(8)-\mathrm{C}(21)$ & $1.31(3)$ \\
\hline $\mathrm{N}(8)-\mathrm{C}(15)$ & $1.52(3)$ \\
\hline $\mathrm{C}(1)-\mathrm{C}(2)$ & $1.40(4)$ \\
\hline $\mathrm{C}(1)-\mathrm{C}(6)$ & $1.45(4)$ \\
\hline $\mathrm{C}(2)-\mathrm{C}(3)$ & $1.21(4)$ \\
\hline $\mathrm{C}(3)-\mathrm{C}(4)$ & $1.49(4)$ \\
\hline$C(4)-C(5)$ & $1.42(4)$ \\
\hline$C(5)-C(6)$ & $1.35(4)$ \\
\hline$C(7)-C(8)$ & $1.57(3)$ \\
\hline$C(9)-C(10)$ & $1.30(3)$ \\
\hline
\end{tabular}




\begin{tabular}{|c|c|}
\hline$C(9)-C(14)$ & $1.45(4)$ \\
\hline$C(10)-C(11)$ & $1.43(4)$ \\
\hline$C(11)-C(12)$ & $1.30(4)$ \\
\hline$C(11)-C(17)$ & $1.57(3)$ \\
\hline $\mathrm{C}(12)-\mathrm{C}(13)$ & $1.48(4)$ \\
\hline$C(13)-C(14)$ & $1.42(4)$ \\
\hline$C(15)-C(20)$ & $1.36(4)$ \\
\hline$C(15)-C(16)$ & $1.46(3)$ \\
\hline$C(16)-C(17)$ & $1.33(4)$ \\
\hline $\mathrm{C}(17)-\mathrm{C}(18)$ & $1.40(4)$ \\
\hline $\mathrm{C}(18)-\mathrm{C}(19)$ & $1.36(4)$ \\
\hline$C(19)-C(20)$ & $1.30(3)$ \\
\hline$C(21)-C(22)$ & $1.49(3)$ \\
\hline$C(23)-C(28)$ & $1.36(4)$ \\
\hline$C(23)-C(24)$ & $1.43(3)$ \\
\hline$C(24)-C(25)$ & $1.38(4)$ \\
\hline$C(25)-C(26)$ & $1.31(4)$ \\
\hline$C(26)-C(27)$ & $1.47(4)$ \\
\hline $\mathrm{C}(27)-\mathrm{C}(28)$ & $1.48(3)$ \\
\hline N11-C11 & $1.324(7)$ \\
\hline N11-C51 & $1.332(7)$ \\
\hline N21-C101 & $1.324(7)$ \\
\hline N21-C61 & $1.324(7)$ \\
\hline C11-C21 & $1.359(7)$ \\
\hline C21-C31 & $1.360(7)$ \\
\hline
\end{tabular}




\begin{tabular}{|c|c|}
\hline C31-C41 & $1.369(7)$ \\
\hline C41-C51 & $1.368(7)$ \\
\hline C51-C61 & $1.468(7)$ \\
\hline C61-C71 & $1.360(7)$ \\
\hline C71-C81 & $1.365(7)$ \\
\hline C81-C91 & $1.356(7)$ \\
\hline C91-C101 & $1.364(7)$ \\
\hline N12-C12 & $1.334(6)$ \\
\hline N12-C52 & $1.342(6)$ \\
\hline $\mathrm{N} 22-\mathrm{C} 102$ & $1.334(6)$ \\
\hline N22-C62 & $1.334(6)$ \\
\hline $\mathrm{C} 12-\mathrm{C} 22$ & $1.370(6)$ \\
\hline $\mathrm{C} 22-\mathrm{C} 32$ & $1.371(6)$ \\
\hline $\mathrm{C} 32-\mathrm{C} 42$ & $1.380(6)$ \\
\hline C42-C52 & $1.379(6)$ \\
\hline C52-C62 & $1.480(7)$ \\
\hline $\mathrm{C} 62-\mathrm{C} 72$ & $1.371(6)$ \\
\hline C72-C82 & $1.376(6)$ \\
\hline C82-C92 & $1.367(6)$ \\
\hline C92-C102 & $1.375(6)$ \\
\hline N13-C13 & $1.322(6)$ \\
\hline N13-C53 & $1.330(6)$ \\
\hline $\mathrm{N} 23-\mathrm{C} 103$ & $1.322(6)$ \\
\hline N23-C63 & $1.323(6)$ \\
\hline $\mathrm{C} 13-\mathrm{C} 23$ & $1.357(7)$ \\
\hline
\end{tabular}




\begin{tabular}{|c|c|}
\hline $\mathrm{C} 23-\mathrm{C} 33$ & $1.358(6)$ \\
\hline C33-C43 & $1.368(6)$ \\
\hline $\mathrm{C} 43-\mathrm{C} 53$ & $1.367(6)$ \\
\hline C53-C63 & $1.467(7)$ \\
\hline C63-C73 & $1.358(7)$ \\
\hline C73-C83 & $1.364(6)$ \\
\hline C83-C93 & $1.354(7)$ \\
\hline C93-C103 & $1.363(6)$ \\
\hline N14-C14 & $1.332(6)$ \\
\hline N14-C54 & $1.340(6)$ \\
\hline N24-C104 & $1.332(6)$ \\
\hline N24-C64 & $1.332(6)$ \\
\hline $\mathrm{C} 14-\mathrm{C} 24$ & $1.368(6)$ \\
\hline $\mathrm{C} 24-\mathrm{C} 34$ & $1.368(6)$ \\
\hline C34-C44 & $1.378(6)$ \\
\hline C44-C54 & $1.377(6)$ \\
\hline C54-C64 & $1.477(7)$ \\
\hline C64-C74 & $1.368(6)$ \\
\hline C74-C84 & $1.373(7)$ \\
\hline C84-C94 & $1.364(6)$ \\
\hline C94-C104 & $1.372(6)$ \\
\hline N15-C15 & $1.300(6)$ \\
\hline N15-C55 & $1.307(6)$ \\
\hline $\mathrm{N} 25-\mathrm{C} 105$ & $1.300(6)$ \\
\hline N25-C65 & $1.301(6)$ \\
\hline
\end{tabular}




\begin{tabular}{|c|c|}
\hline $\mathrm{C} 15-\mathrm{C} 25$ & $1.334(6)$ \\
\hline $\mathrm{C} 25-\mathrm{C} 35$ & $1.335(6)$ \\
\hline $\mathrm{C} 35-\mathrm{C} 45$ & $1.345(6)$ \\
\hline $\mathrm{C} 45-\mathrm{C} 55$ & $1.344(6)$ \\
\hline C55-C65 & $1.442(6)$ \\
\hline $\mathrm{C} 65-\mathrm{C} 75$ & $1.335(6)$ \\
\hline C75-C85 & $1.340(6)$ \\
\hline C85-C95 & $1.331(6)$ \\
\hline C95-C105 & $1.339(6)$ \\
\hline N16-C16 & $1.331(5)$ \\
\hline N16-C56 & $1.339(5)$ \\
\hline N26-C106 & $1.331(5)$ \\
\hline N26-C66 & $1.331(5)$ \\
\hline $\mathrm{C} 16-\mathrm{C} 26$ & $1.366(5)$ \\
\hline $\mathrm{C} 26-\mathrm{C} 36$ & $1.367(5)$ \\
\hline $\mathrm{C} 36-\mathrm{C} 46$ & $1.377(5)$ \\
\hline C46-C56 & $1.376(5)$ \\
\hline C56-C66 & $1.476(5)$ \\
\hline C66-C76 & $1.367(5)$ \\
\hline C76-C86 & $1.373(5)$ \\
\hline C86-C96 & $1.364(5)$ \\
\hline C96-C106 & $1.371(5)$ \\
\hline N17-C17 & $1.339(6)$ \\
\hline N17-C57 & $1.346(6)$ \\
\hline N27-C107 & $1.338(6)$ \\
\hline
\end{tabular}




\begin{tabular}{|c|c|}
\hline N27-C67 & $1.339(6)$ \\
\hline $\mathrm{C} 17-\mathrm{C} 27$ & $1.374(6)$ \\
\hline C27-C37 & $1.375(7)$ \\
\hline C37-C47 & $1.384(6)$ \\
\hline C47-C57 & $1.383(6)$ \\
\hline C57-C67 & $1.485(7)$ \\
\hline C67-C77 & $1.375(6)$ \\
\hline C77-C87 & $1.380(6)$ \\
\hline C87-C97 & $1.371(6)$ \\
\hline C97-C107 & $1.379(6)$ \\
\hline N18-C18 & $1.350(6)$ \\
\hline N18-C58 & $1.358(6)$ \\
\hline N28-C68 & $1.350(6)$ \\
\hline N28-C108 & $1.351(6)$ \\
\hline $\mathrm{C} 18-\mathrm{C} 28$ & $1.387(6)$ \\
\hline C28-C38 & $1.387(6)$ \\
\hline C38-C48 & $1.397(6)$ \\
\hline C48-C58 & $1.396(6)$ \\
\hline C58-C68 & $1.498(7)$ \\
\hline C68-C78 & $1.387(6)$ \\
\hline C78-C88 & $1.393(6)$ \\
\hline C88-C98 & $1.383(6)$ \\
\hline C98-C108 & $1.391(6)$ \\
\hline P19-F69 & $1.502(19)$ \\
\hline P19-F39 & $1.533(19)$ \\
\hline
\end{tabular}




\begin{tabular}{|c|c|}
\hline P19-F29 & $1.547(18)$ \\
\hline P19-F59 & $1.585(18)$ \\
\hline P19-F49 & $1.600(19)$ \\
\hline P19-F19 & $1.662(18)$ \\
\hline P110-F610 & $1.502(19)$ \\
\hline P110-F310 & $1.537(18)$ \\
\hline P110-F210 & $1.552(18)$ \\
\hline P110-F510 & $1.591(18)$ \\
\hline P110-F410 & $1.615(18)$ \\
\hline P110-F110 & $1.647(18)$ \\
\hline P111-F611 & $1.494(19)$ \\
\hline P111-F211 & $1.541(18)$ \\
\hline P111-F311 & $1.543(18)$ \\
\hline P111-F511 & $1.578(18)$ \\
\hline P111-F411 & $1.598(18)$ \\
\hline P111-F111 & $1.665(18)$ \\
\hline P112-F612 & $1.49(2)$ \\
\hline P112-F212 & $1.54(2)$ \\
\hline P112-F312 & $1.542(19)$ \\
\hline P112-F512 & $1.584(19)$ \\
\hline P112-F412 & $1.606(19)$ \\
\hline P112-F112 & $1.67(2)$ \\
\hline O113-C213 & $1.21(4)$ \\
\hline C113-C213 & $1.43(4)$ \\
\hline C213-C 313 & $1.70(4)$ \\
\hline
\end{tabular}




$\begin{array}{lc}\mathrm{O} 114-\mathrm{C} 214 & 1.21(4) \\ \mathrm{C} 114-\mathrm{C} 214 & 1.43(4) \\ \mathrm{C} 214-\mathrm{C} 314 & 1.70(4) \\ \mathrm{O} 115-\mathrm{C} 215 & 1.21(4) \\ \mathrm{C} 115-\mathrm{C} 215 & 1.43(4) \\ \mathrm{C} 215-\mathrm{C} 315 & 1.70(4)\end{array}$

N22-Ru(1)-N11

$\mathrm{N} 22-\mathrm{Ru}(1)-\mathrm{N} 12$

N11-Ru(1)-N12

$\mathrm{N} 22-\mathrm{Ru}(1)-\mathrm{N} 21$

N11-Ru(1)-N21

$\mathrm{N} 12-\mathrm{Ru}(1)-\mathrm{N} 21$

$\mathrm{N} 22-\mathrm{Ru}(1)-\mathrm{N}(2)$

$\mathrm{N} 11-\mathrm{Ru}(1)-\mathrm{N}(2)$

$\mathrm{N} 12-\mathrm{Ru}(1)-\mathrm{N}(2)$

$\mathrm{N} 21-\mathrm{Ru}(1)-\mathrm{N}(2)$

N22-Ru(1)-N(1)

N11-Ru(1)-N(1)

N12-Ru(1)-N(1)

$\mathrm{N} 21-\mathrm{Ru}(1)-\mathrm{N}(1)$

$\mathrm{N}(2)-\mathrm{Ru}(1)-\mathrm{N}(1)$

$\mathrm{N} 24-\mathrm{Ru}(2)-\mathrm{N} 13$

$\mathrm{N} 24-\mathrm{Ru}(2)-\mathrm{N} 23$

N13-Ru(2)-N23
178.6(7)

81.7(7)

97.1(6)

99.7(7)

79.7(7)

96.1(6)

86.6(10)

94.1(10)

93.7(9)

169.0(9)

96.3(8)

$85.0(8)$

172.0(8)

91.9(8)

78.4(10)

173.5(6)

93.5(6)

$80.0(7)$ 


\begin{tabular}{|c|c|}
\hline N24-Ru(2)-N14 & $82.6(7)$ \\
\hline N13-Ru(2)-N14 & $97.3(6)$ \\
\hline $\mathrm{N} 23-\mathrm{Ru}(2)-\mathrm{N} 14$ & $99.6(6)$ \\
\hline N24-Ru(2)-N(4) & $91.6(9)$ \\
\hline N13-Ru(2)-N(4) & $89.8(9)$ \\
\hline N23-Ru(2)-N(4) & $91.8(8)$ \\
\hline N14-Ru(2)-N(4) & $167.5(8)$ \\
\hline N24-Ru(2)-N(3) & $90.0(9)$ \\
\hline N13-Ru(2)-N(3) & $96.5(9)$ \\
\hline N23-Ru(2)-N(3) & $174.0(9)$ \\
\hline N14-Ru(2)-N(3) & $85.6(8)$ \\
\hline $\mathrm{N}(4)-\mathrm{Ru}(2)-\mathrm{N}(3)$ & $83.3(11)$ \\
\hline $\mathrm{N} 25-\mathrm{Ru}(3)-\mathrm{N} 15$ & $78.8(5)$ \\
\hline N25-Ru(3)-N16 & $95.8(5)$ \\
\hline N15-Ru(3)-N16 & $100.9(5)$ \\
\hline $\mathrm{N} 25-\mathrm{Ru}(3)-\mathrm{N} 26$ & $174.2(5)$ \\
\hline N15-Ru(3)-N26 & $98.2(5)$ \\
\hline N16-Ru(3)-N26 & $79.8(5)$ \\
\hline N25-Ru(3)-N(5) & $92.2(8)$ \\
\hline $\mathrm{N} 15-\mathrm{Ru}(3)-\mathrm{N}(5)$ & $88.1(8)$ \\
\hline N16-Ru(3)-N(5) & $168.9(7)$ \\
\hline $\mathrm{N} 26-\mathrm{Ru}(3)-\mathrm{N}(5)$ & $92.6(7)$ \\
\hline N25-Ru(3)-N(6) & $93.3(8)$ \\
\hline N15-Ru(3)-N(6) & $168.7(8)$ \\
\hline N16-Ru(3)-N(6) & $87.9(7)$ \\
\hline
\end{tabular}




\begin{tabular}{|c|c|}
\hline N26-Ru(3)-N(6) & $90.4(7)$ \\
\hline $\mathrm{N}(5)-\mathrm{Ru}(3)-\mathrm{N}(6)$ & $84.1(9)$ \\
\hline N18-Ru(4)-N27 & $98.7(6)$ \\
\hline $\mathrm{N} 18-\mathrm{Ru}(4)-\mathrm{N} 28$ & $83.9(6)$ \\
\hline $\mathrm{N} 27-\mathrm{Ru}(4)-\mathrm{N} 28$ & $97.3(6)$ \\
\hline N18-Ru(4)-N(8) & $91.9(8)$ \\
\hline $\mathrm{N} 27-\mathrm{Ru}(4)-\mathrm{N}(8)$ & $167.4(8)$ \\
\hline N28-Ru(4)-N(8) & $90.6(8)$ \\
\hline N18-Ru(4)-N(7) & $170.7(8)$ \\
\hline $\mathrm{N} 27-\mathrm{Ru}(4)-\mathrm{N}(7)$ & $90.6(8)$ \\
\hline $\mathrm{N} 28-\mathrm{Ru}(4)-\mathrm{N}(7)$ & $95.2(8)$ \\
\hline $\mathrm{N}(8)-\mathrm{Ru}(4)-\mathrm{N}(7)$ & $78.9(10)$ \\
\hline N18-Ru(4)-N17 & $95.5(6)$ \\
\hline N27-Ru(4)-N17 & $80.9(6)$ \\
\hline N28-Ru(4)-N17 & $178.0(6)$ \\
\hline $\mathrm{N}(8)-\mathrm{Ru}(4)-\mathrm{N} 17$ & $91.3(8)$ \\
\hline $\mathrm{N}(7)-\mathrm{Ru}(4)-\mathrm{N} 17$ & $85.7(8)$ \\
\hline $\mathrm{C}(8)-\mathrm{N}(1)-\mathrm{C}(14)$ & 101(2) \\
\hline $\mathrm{C}(8)-\mathrm{N}(1)-\mathrm{Ru}(1)$ & 111.2(19) \\
\hline $\mathrm{C}(14)-\mathrm{N}(1)-\mathrm{Ru}(1)$ & $148(2)$ \\
\hline $\mathrm{C}(7)-\mathrm{N}(2)-\mathrm{C}(1)$ & $100(2)$ \\
\hline $\mathrm{C}(7)-\mathrm{N}(2)-\mathrm{Ru}(1)$ & $116(2)$ \\
\hline $\mathrm{C}(1)-\mathrm{N}(2)-\mathrm{Ru}(1)$ & $144(2)$ \\
\hline $\mathrm{C}(6)-\mathrm{N}(3)-\mathrm{C}(7)$ & $104(2)$ \\
\hline $\mathrm{C}(6)-\mathrm{N}(3)-\mathrm{Ru}(2)$ & $149(3)$ \\
\hline
\end{tabular}




\begin{tabular}{|c|c|}
\hline $\mathrm{C}(7)-\mathrm{N}(3)-\mathrm{Ru}(2)$ & $103.1(18)$ \\
\hline $\mathrm{C}(8)-\mathrm{N}(4)-\mathrm{C}(9)$ & $104(2)$ \\
\hline $\mathrm{C}(8)-\mathrm{N}(4)-\mathrm{Ru}(2)$ & $109(2)$ \\
\hline $\mathrm{C}(9)-\mathrm{N}(4)-\mathrm{Ru}(2)$ & $140(2)$ \\
\hline $\mathrm{C}(21)-\mathrm{N}(5)-\mathrm{C}(20)$ & $103(2)$ \\
\hline $\mathrm{C}(21)-\mathrm{N}(5)-\mathrm{Ru}(3)$ & $107.1(18)$ \\
\hline $\mathrm{C}(20)-\mathrm{N}(5)-\mathrm{Ru}(3)$ & $150(2)$ \\
\hline $\mathrm{C}(23)-\mathrm{N}(6)-\mathrm{C}(22)$ & $100(2)$ \\
\hline $\mathrm{C}(23)-\mathrm{N}(6)-\mathrm{Ru}(3)$ & $154(2)$ \\
\hline $\mathrm{C}(22)-\mathrm{N}(6)-\mathrm{Ru}(3)$ & $105.3(17)$ \\
\hline $\mathrm{C}(22)-\mathrm{N}(7)-\mathrm{C}(28)$ & $98(2)$ \\
\hline $\mathrm{C}(22)-\mathrm{N}(7)-\mathrm{Ru}(4)$ & $112.1(19)$ \\
\hline $\mathrm{C}(28)-\mathrm{N}(7)-\mathrm{Ru}(4)$ & $148(2)$ \\
\hline $\mathrm{C}(21)-\mathrm{N}(8)-\mathrm{C}(15)$ & $96.0(19)$ \\
\hline $\mathrm{C}(21)-\mathrm{N}(8)-\mathrm{Ru}(4)$ & $116.9(18)$ \\
\hline $\mathrm{C}(15)-\mathrm{N}(8)-\mathrm{Ru}(4)$ & $146.6(18)$ \\
\hline$C(2)-C(1)-C(6)$ & $119(3)$ \\
\hline $\mathrm{C}(2)-\mathrm{C}(1)-\mathrm{N}(2)$ & $134(3)$ \\
\hline $\mathrm{C}(6)-\mathrm{C}(1)-\mathrm{N}(2)$ & $105.5(16)$ \\
\hline$C(3)-C(2)-C(1)$ & $123(4)$ \\
\hline$C(2)-C(3)-C(4)$ & $117(3)$ \\
\hline$C(5)-C(4)-C(3)$ & $119(3)$ \\
\hline$C(6)-C(5)-C(4)$ & $117(3)$ \\
\hline $\mathrm{N}(3)-\mathrm{C}(6)-\mathrm{C}(5)$ & $131(3)$ \\
\hline $\mathrm{N}(3)-\mathrm{C}(6)-\mathrm{C}(1)$ & $109.6(18)$ \\
\hline
\end{tabular}




\begin{tabular}{|c|c|}
\hline$C(5)-C(6)-C(1)$ & $119(3)$ \\
\hline $\mathrm{N}(2)-\mathrm{C}(7)-\mathrm{N}(3)$ & $120(2)$ \\
\hline $\mathrm{N}(2)-\mathrm{C}(7)-\mathrm{C}(8)$ & $118(3)$ \\
\hline $\mathrm{N}(3)-\mathrm{C}(7)-\mathrm{C}(8)$ & $120(3)$ \\
\hline $\mathrm{N}(4)-\mathrm{C}(8)-\mathrm{N}(1)$ & $122(2)$ \\
\hline $\mathrm{N}(4)-\mathrm{C}(8)-\mathrm{C}(7)$ & $121(3)$ \\
\hline $\mathrm{N}(1)-\mathrm{C}(8)-\mathrm{C}(7)$ & $116(2)$ \\
\hline $\mathrm{C}(10)-\mathrm{C}(9)-\mathrm{N}(4)$ & $136(3)$ \\
\hline $\mathrm{C}(10)-\mathrm{C}(9)-\mathrm{C}(14)$ & $122(3)$ \\
\hline $\mathrm{N}(4)-\mathrm{C}(9)-\mathrm{C}(14)$ & $101.5(17)$ \\
\hline $\mathrm{C}(9)-\mathrm{C}(10)-\mathrm{C}(11)$ & $118(3)$ \\
\hline $\mathrm{C}(12)-\mathrm{C}(11)-\mathrm{C}(10)$ & $122(3)$ \\
\hline $\mathrm{C}(12)-\mathrm{C}(11)-\mathrm{C}(17)$ & $121(3)$ \\
\hline $\mathrm{C}(10)-\mathrm{C}(11)-\mathrm{C}(17)$ & $118(3)$ \\
\hline $\mathrm{C}(11)-\mathrm{C}(12)-\mathrm{C}(13)$ & $123(3)$ \\
\hline$C(14)-C(13)-C(12)$ & $112(3)$ \\
\hline $\mathrm{N}(1)-\mathrm{C}(14)-\mathrm{C}(13)$ & $125(3)$ \\
\hline $\mathrm{N}(1)-\mathrm{C}(14)-\mathrm{C}(9)$ & $108.4(17)$ \\
\hline $\mathrm{C}(13)-\mathrm{C}(14)-\mathrm{C}(9)$ & $119(3)$ \\
\hline$C(20)-C(15)-C(16)$ & $123(2)$ \\
\hline $\mathrm{C}(20)-\mathrm{C}(15)-\mathrm{N}(8)$ & $109.2(13)$ \\
\hline $\mathrm{C}(16)-\mathrm{C}(15)-\mathrm{N}(8)$ & $127(3)$ \\
\hline$C(17)-C(16)-C(15)$ & $112(3)$ \\
\hline$C(16)-C(17)-C(18)$ & $122(3)$ \\
\hline$C(16)-C(17)-C(11)$ & $116(3)$ \\
\hline
\end{tabular}




\begin{tabular}{|c|c|}
\hline $\mathrm{C}(18)-\mathrm{C}(17)-\mathrm{C}(11)$ & $122(3)$ \\
\hline $\mathrm{C}(19)-\mathrm{C}(18)-\mathrm{C}(17)$ & $122(3)$ \\
\hline $\mathrm{C}(20)-\mathrm{C}(19)-\mathrm{C}(18)$ & $118(3)$ \\
\hline $\mathrm{C}(19)-\mathrm{C}(20)-\mathrm{N}(5)$ & $130(3)$ \\
\hline$C(19)-C(20)-C(15)$ & $121(3)$ \\
\hline N(5)-C(20)-C(15) & $109.2(15)$ \\
\hline $\mathrm{N}(8)-\mathrm{C}(21)-\mathrm{N}(5)$ & $122(2)$ \\
\hline $\mathrm{N}(8)-\mathrm{C}(21)-\mathrm{C}(22)$ & $112(2)$ \\
\hline $\mathrm{N}(5)-\mathrm{C}(21)-\mathrm{C}(22)$ & $124(2)$ \\
\hline $\mathrm{N}(7)-\mathrm{C}(22)-\mathrm{N}(6)$ & $120(2)$ \\
\hline $\mathrm{N}(7)-\mathrm{C}(22)-\mathrm{C}(21)$ & $120(2)$ \\
\hline $\mathrm{N}(6)-\mathrm{C}(22)-\mathrm{C}(21)$ & $119(2)$ \\
\hline $\mathrm{N}(6)-\mathrm{C}(23)-\mathrm{C}(28)$ & $113.2(17)$ \\
\hline $\mathrm{N}(6)-\mathrm{C}(23)-\mathrm{C}(24)$ & $126(3)$ \\
\hline $\mathrm{C}(28)-\mathrm{C}(23)-\mathrm{C}(24)$ & $120(2)$ \\
\hline$C(25)-C(24)-C(23)$ & $119(3)$ \\
\hline$C(26)-C(25)-C(24)$ & $121(3)$ \\
\hline$C(25)-C(26)-C(27)$ & $124(3)$ \\
\hline $\mathrm{C}(26)-\mathrm{C}(27)-\mathrm{C}(28)$ & $112(3)$ \\
\hline $\mathrm{C}(23)-\mathrm{C}(28)-\mathrm{N}(7)$ & $108.2(15)$ \\
\hline $\mathrm{C}(23)-\mathrm{C}(28)-\mathrm{C}(27)$ & $122(2)$ \\
\hline $\mathrm{N}(7)-\mathrm{C}(28)-\mathrm{C}(27)$ & $130(3)$ \\
\hline C11-N11-C51 & 119.2 \\
\hline C11-N11-Ru(1) & $127.2(6)$ \\
\hline C51-N11-Ru(1) & $112.4(6)$ \\
\hline
\end{tabular}




\begin{tabular}{|c|c|}
\hline C101-N21-C61 & 118.9 \\
\hline C101-N21-Ru(1) & $127.4(6)$ \\
\hline C61-N21-Ru(1) & $112.9(6)$ \\
\hline N11-C11-C21 & 122.4 \\
\hline C11-C21-C31 & 119.0 \\
\hline C21-C31-C41 & 119.0 \\
\hline C51-C41-C31 & 119.5 \\
\hline N11-C51-C41 & 120.9 \\
\hline N11-C51-C61 & 117.2 \\
\hline C41-C51-C61 & 121.9 \\
\hline N21-C61-C71 & 121.3 \\
\hline N21-C61-C51 & 115.9 \\
\hline C71-C61-C51 & 122.8 \\
\hline C61-C71-C81 & 119.6 \\
\hline C91-C81-C71 & 119.3 \\
\hline C81-C91-C101 & 118.2 \\
\hline N21-C101-C91 & 122.8 \\
\hline C12-N12-C52 & 119.2 \\
\hline C12-N12-Ru(1) & $129.3(5)$ \\
\hline C52-N12-Ru(1) & $111.4(5)$ \\
\hline C102-N22-C62 & 118.8 \\
\hline C102-N22-Ru(1) & $127.5(5)$ \\
\hline C62-N22-Ru(1) & $113.3(5)$ \\
\hline N12-C12-C22 & 122.4 \\
\hline C12-C22-C32 & 119.0 \\
\hline
\end{tabular}




\begin{tabular}{|c|c|}
\hline $\mathrm{C} 22-\mathrm{C} 32-\mathrm{C} 42$ & 119.0 \\
\hline C52-C42-C32 & 119.5 \\
\hline N12-C52-C42 & 120.9 \\
\hline N12-C52-C62 & 117.2 \\
\hline C42-C52-C62 & 121.9 \\
\hline N22-C62-C72 & 121.3 \\
\hline N22-C62-C52 & 115.9 \\
\hline C72-C62-C52 & 122.8 \\
\hline C62-C72-C82 & 119.6 \\
\hline C92-C82-C72 & 119.3 \\
\hline C82-C92-C102 & 118.2 \\
\hline N22-C102-C92 & 122.8 \\
\hline C13-N13-C53 & 119.2 \\
\hline C13-N13-Ru(2) & $127.4(5)$ \\
\hline $\mathrm{C} 53-\mathrm{N} 13-\mathrm{Ru}(2)$ & $112.6(5)$ \\
\hline C103-N23-C63 & 118.9 \\
\hline C103-N23-Ru(2) & $127.5(5)$ \\
\hline C63-N23-Ru(2) & $113.2(5)$ \\
\hline N13-C13-C23 & 122.4 \\
\hline $\mathrm{C} 13-\mathrm{C} 23-\mathrm{C} 33$ & 119.0 \\
\hline C23-C33-C43 & 119.0 \\
\hline C53-C43-C33 & 119.4 \\
\hline N13-C53-C43 & 121.0 \\
\hline N13-C53-C63 & 117.2 \\
\hline C43-C53-C63 & 121.8 \\
\hline
\end{tabular}




\begin{tabular}{|c|c|}
\hline N23-C63-C73 & 121.3 \\
\hline N23-C63-C53 & 115.9 \\
\hline C73-C63-C53 & 122.8 \\
\hline C63-C73-C83 & 119.6 \\
\hline C93-C83-C73 & 119.3 \\
\hline C83-C93-C103 & 118.2 \\
\hline N23-C103-C93 & 122.7 \\
\hline C14-N14-C54 & 119.3 \\
\hline C14-N14-Ru(2) & $130.9(5)$ \\
\hline C54-N14-Ru(2) & $109.4(5)$ \\
\hline C104-N24-C64 & 118.9 \\
\hline C104-N24-Ru(2) & $126.6(5)$ \\
\hline C64-N24-Ru(2) & $114.4(5)$ \\
\hline N14-C14-C24 & 122.4 \\
\hline C14-C24-C34 & 119.0 \\
\hline C24-C34-C44 & 119.0 \\
\hline C54-C44-C34 & 119.4 \\
\hline N14-C54-C44 & 120.9 \\
\hline N14-C54-C64 & 117.2 \\
\hline C44-C54-C64 & 121.9 \\
\hline N24-C64-C74 & 121.3 \\
\hline N24-C64-C54 & 115.9 \\
\hline C74-C64-C54 & 122.8 \\
\hline C64-C74-C84 & 119.6 \\
\hline C94-C84-C74 & 119.3 \\
\hline
\end{tabular}




\begin{tabular}{|c|c|}
\hline C84-C94-C104 & 118.2 \\
\hline N24-C104-C94 & 122.8 \\
\hline C15-N15-C55 & 119.2 \\
\hline C15-N15-Ru(3) & $127.9(4)$ \\
\hline C55-N15-Ru(3) & $112.9(4)$ \\
\hline C105-N25-C65 & 118.9 \\
\hline $\mathrm{C} 105-\mathrm{N} 25-\mathrm{Ru}(3)$ & $126.0(4)$ \\
\hline $\mathrm{C} 65-\mathrm{N} 25-\mathrm{Ru}(3)$ & $115.0(4)$ \\
\hline N15-C15-C25 & 122.4 \\
\hline $\mathrm{C} 15-\mathrm{C} 25-\mathrm{C} 35$ & 119.0 \\
\hline C25-C35-C45 & 119.0 \\
\hline C55-C45-C35 & 119.4 \\
\hline N15-C55-C45 & 120.9 \\
\hline N15-C55-C65 & 117.2 \\
\hline C45-C55-C65 & 121.8 \\
\hline N25-C65-C75 & 121.3 \\
\hline N25-C65-C55 & 115.9 \\
\hline C75-C65-C55 & 122.8 \\
\hline C65-C75-C85 & 119.6 \\
\hline C95-C85-C75 & 119.3 \\
\hline C85-C95-C105 & 118.2 \\
\hline N25-C105-C95 & 122.8 \\
\hline C16-N16-C56 & 119.2 \\
\hline C16-N16-Ru(3) & $127.9(4)$ \\
\hline $\mathrm{C} 56-\mathrm{N} 16-\mathrm{Ru}(3)$ & $112.4(4)$ \\
\hline
\end{tabular}




\begin{tabular}{|c|c|}
\hline C106-N26-C66 & 118.9 \\
\hline C106-N26-Ru(3) & $127.2(4)$ \\
\hline C66-N26-Ru(3) & $113.1(4)$ \\
\hline N16-C16-C26 & 122.4 \\
\hline $\mathrm{C} 16-\mathrm{C} 26-\mathrm{C} 36$ & 118.9 \\
\hline $\mathrm{C} 26-\mathrm{C} 36-\mathrm{C} 46$ & 119.0 \\
\hline C56-C46-C36 & 119.5 \\
\hline N16-C56-C46 & 120.9 \\
\hline N16-C56-C66 & 117.2 \\
\hline C46-C56-C66 & 121.9 \\
\hline N26-C66-C76 & 121.3 \\
\hline N26-C66-C56 & 115.9 \\
\hline C76-C66-C56 & 122.8 \\
\hline C66-C76-C86 & 119.6 \\
\hline C96-C86-C76 & 119.3 \\
\hline C86-C96-C106 & 118.2 \\
\hline N26-C106-C96 & 122.8 \\
\hline C17-N17-C57 & 119.2 \\
\hline C17-N17-Ru(4) & $129.6(5)$ \\
\hline C57-N17-Ru(4) & $110.5(5)$ \\
\hline C107-N27-C67 & 118.9 \\
\hline C107-N27-Ru(4) & $126.1(5)$ \\
\hline C67-N27-Ru(4) & $114.7(5)$ \\
\hline $\mathrm{N} 17-\mathrm{C} 17-\mathrm{C} 27$ & 122.4 \\
\hline C17-C27-C37 & 119.0 \\
\hline
\end{tabular}




\begin{tabular}{|c|c|}
\hline C27-C37-C47 & 119.0 \\
\hline C57-C47-C37 & 119.5 \\
\hline N17-C57-C47 & 120.9 \\
\hline N17-C57-C67 & 117.2 \\
\hline C47-C57-C67 & 121.9 \\
\hline N27-C67-C77 & 121.3 \\
\hline N27-C67-C57 & 115.9 \\
\hline C77-C67-C57 & 122.8 \\
\hline C67-C77-C87 & 119.6 \\
\hline C97-C87-C77 & 119.3 \\
\hline C87-C97-C107 & 118.2 \\
\hline N27-C107-C97 & 122.8 \\
\hline C18-N18-C58 & 119.3 \\
\hline C18-N18-Ru(4) & $129.6(5)$ \\
\hline C58-N18-Ru(4) & $111.0(5)$ \\
\hline C68-N28-C108 & 118.8 \\
\hline C68-N28-Ru(4) & $111.5(5)$ \\
\hline C108-N28-Ru(4) & $129.4(5)$ \\
\hline N18-C18-C28 & 122.4 \\
\hline C18-C28-C38 & 118.9 \\
\hline C28-C38-C48 & 119.0 \\
\hline C58-C48-C38 & 119.5 \\
\hline N18-C58-C48 & 120.9 \\
\hline N18-C58-C68 & 117.2 \\
\hline C48-C58-C68 & 121.9 \\
\hline
\end{tabular}




\begin{tabular}{|c|c|}
\hline N28-C68-C78 & 121.3 \\
\hline N28-C68-C58 & 115.9 \\
\hline C78-C68-C58 & 122.8 \\
\hline C68-C78-C88 & 119.6 \\
\hline C98-C88-C78 & 119.3 \\
\hline C88-C98-C108 & 118.2 \\
\hline N28-C108-C98 & 122.7 \\
\hline F69-P19-F39 & $90.3(16)$ \\
\hline F69-P19-F29 & $87.8(13)$ \\
\hline F39-P19-F29 & $94.8(14)$ \\
\hline F69-P19-F59 & $177.6(15)$ \\
\hline F39-P19-F59 & $89.5(15)$ \\
\hline F29-P19-F59 & $89.9(14)$ \\
\hline F69-P19-F49 & $91.6(16)$ \\
\hline F39-P19-F49 & $176.9(16)$ \\
\hline F29-P19-F49 & $82.8(13)$ \\
\hline F59-P19-F49 & $88.6(14)$ \\
\hline F69-P19-F19 & $96.7(14)$ \\
\hline F39-P19-F19 & $88.0(14)$ \\
\hline F29-P19-F19 & $174.7(15)$ \\
\hline F59-P19-F19 & $85.6(12)$ \\
\hline F49-P19-F19 & $94.2(13)$ \\
\hline F610-P110-F310 & $90.9(13)$ \\
\hline F610-P110-F210 & $88.6(14)$ \\
\hline F310-P110-F210 & $93.1(12)$ \\
\hline
\end{tabular}




\begin{tabular}{|c|c|}
\hline F610-P110-F510 & $177.1(17)$ \\
\hline F310-P110-F510 & $91.3(14)$ \\
\hline F210-P110-F510 & $89.4(13)$ \\
\hline F610-P110-F410 & $90.2(14)$ \\
\hline F310-P110-F410 & $177.0(14)$ \\
\hline F210-P110-F410 & $84.1(12)$ \\
\hline F510-P110-F410 & $87.5(12)$ \\
\hline F610-P110-F110 & $95.6(14)$ \\
\hline F310-P110-F110 & $90.4(13)$ \\
\hline F210-P110-F110 & $174.5(14)$ \\
\hline F510-P110-F110 & $86.3(13)$ \\
\hline F410-P110-F110 & $92.2(12)$ \\
\hline F611-P111-F211 & $89.6(14)$ \\
\hline F611-P111-F311 & $92.3(14)$ \\
\hline F211-P111-F311 & $92.3(14)$ \\
\hline F611-P111-F511 & $178.1(16)$ \\
\hline F211-P111-F511 & $91.6(13)$ \\
\hline F311-P111-F511 & $89.0(13)$ \\
\hline F611-P111-F411 & $90.7(14)$ \\
\hline F211-P111-F411 & $85.3(12)$ \\
\hline F311-P111-F411 & $176.1(15)$ \\
\hline F511-P111-F411 & $88.0(13)$ \\
\hline F611-P111-F111 & $93.2(15)$ \\
\hline F211-P111-F111 & $176.9(17)$ \\
\hline F311-P111-F111 & $88.9(13)$ \\
\hline
\end{tabular}




\begin{tabular}{|c|c|}
\hline F511-P111-F111 & $85.6(14)$ \\
\hline F411-P111-F111 & $93.4(14)$ \\
\hline F612-P112-F212 & $89.7(17)$ \\
\hline F612-P112-F312 & $92.0(16)$ \\
\hline F212-P112-F312 & $95.4(15)$ \\
\hline F612-P112-F512 & $180(2)$ \\
\hline F212-P112-F512 & $89.9(15)$ \\
\hline F312-P112-F512 & $88.2(15)$ \\
\hline F612-P112-F412 & $91.3(16)$ \\
\hline F212-P112-F412 & $83.9(14)$ \\
\hline F312-P112-F412 & $176.7(18)$ \\
\hline F512-P112-F412 & $88.6(14)$ \\
\hline F612-P112-F112 & $94.7(17)$ \\
\hline F212-P112-F112 & $174.6(17)$ \\
\hline F312-P112-F112 & $87.6(15)$ \\
\hline F512-P112-F112 & $85.7(15)$ \\
\hline F412-P112-F112 & $92.9(14)$ \\
\hline O113-C213-C113 & $130(3)$ \\
\hline $\mathrm{O} 113-\mathrm{C} 213-\mathrm{C} 313$ & $116(3)$ \\
\hline C113-C213-C313 & $114(3)$ \\
\hline O114-C214-C114 & $130(3)$ \\
\hline O114-C214-C314 & $116(3)$ \\
\hline C114-C214-C314 & $114(3)$ \\
\hline $\mathrm{O} 115-\mathrm{C} 215-\mathrm{C} 115$ & $130(3)$ \\
\hline O115-C215-C315 & $116(3)$ \\
\hline
\end{tabular}


